\title{
Wnt10B is critical for the progression of gastric cancer
}

\author{
XIAO-DAN WU ${ }^{1 *}$, QING-LI BIE ${ }^{2 *}$, BIN ZHANG $^{3}$, ZI-HE YAN $^{1}$ and ZHI-JUN HAN ${ }^{1}$ \\ ${ }^{1}$ Department of Laboratory Medicine, Nanjing Medical University Affiliated Wuxi Second Hospital, Wuxi, Jiangsu 214002; \\ ${ }^{2}$ The Key Laboratory Medicine of Jiangsu Province, School of Medicine, Jiangsu University, Zhenjiang, Jiangsu 212013, \\ ${ }^{3}$ Department of Laboratory Medicine, Affiliated Hospital of Jining Medical University, Jining, Shandong 272000, P.R. China
}

Received February 12, 2016; Accepted February 1, 2017

DOI: $10.3892 / 01.2017 .5992$

\begin{abstract}
The family of Wnt proteins have been implicated in embryogenesis by regulation of cell fate and pattern formation, and also in human carcinogenesis. Wnt10B was previously shown to be involved in breast cancer development. The present study assessed the association of Wnt10B expression in human gastric cancer tissue specimens with clinicopathological data from these patients. Wnt10B expression in the regulation of gastric cancer cell proliferation and migration capacity in vitro was then investigated. The data revealed that Wnt10B mRNA and protein were upregulated in gastric cancer tissue samples and the upregulated Wnt10B mRNA was associated with gastric cancer metastasizing to lymph nodes. Knockdown of Wnt10B expression reduced gastric cancer cell proliferation and migration, as well as expression of a cell proliferation marker Ki67. Knockdown of Wnt10B expression inhibited tumor cell epithelial-mesenchymal transition by upregulation of E-cadherin and downregulation of $\mathrm{N}$-cadherin. In addition, Wnt10B knockdown also suppressed tumor cell stemness by downregulation of octamer-binding transcription factor 4 and Nanog expression. The present data indicated that Wnt10B expression performs an important role in gastric cancer progression in vitro. Therefore, targeting of Wnt10B expression or activity may be investigated as a possible strategy for the control of gastric cancer.
\end{abstract}

\section{Introduction}

Gastric cancer is the second most common cancer in the world, accounting for one million cancer-associated mortalities annually and representing a major worldwide public health problem (1). With advances in early detection,

Correspondence to: Dr Zhi-Jun Han, Department of Laboratory Medicine, Nanjing Medical University Affiliated Wuxi Second Hospital, 68 Zhongshan Road, Wuxi, Jiangsu 214002, P.R. China E-mail: zjhan1125@163.com

\section{${ }^{*}$ Contributed equally}

Key words: Wnt10B, gastric cancer, cancer metastasis, epithelial-mesenchymal transition, stemness standardized surgical treatment and improved perioperative care, the outcomes of patients with gastric cancer have notably improved (2). However, diagnosis of gastric cancer at early stages remains a challenge in clinical settings due to asymptomatic or nonspecific symptoms in early lesions, as well as lack of effective biomarkers. Gastric cancer development and progression involves multiple factors (3). Helicobacter pylori infection is a major risk factor in gastric cancer development (4). Tobacco smoke, alcohol consumption and dietary factors, including smoked foods, salt-rich foods, red meat and pickled vegetables are also associated with increased gastric cancer incidence (5). These factors potentially involve chronic inflammation and gastric carcinogenesis (5). Over the past decades, efforts have been made to elucidate the underlying mechanisms and to identify novel diagnostic biomarkers and therapeutic targets for gastric cancer. However, gastric cancer mortality remains high (6). Therefore, additional investigation and attention is required to identify novel biomarkers for early diagnosis, prognosis and treatment response prediction of gastric cancer and/or to identify novel targets to control gastric cancer.

Nusse and Varmus identified the first member of the Wnt gene family in 1982 (7), and it is now well accepted that these highly conserved Wnt secreted proteins are critical mediators of cell-to-cell signaling, cell fate and pattern formation during embryonic development. The Wnt family of proteins includes at least 19 secreted-type glycoproteins with 22-24 conserved cysteine residues (8). This canonical Wnt pathway regulates target gene expression in the nucleus to control cell proliferation (9), and the non-canonical pathways regulate numerous other aspects of cell biology, including cell motility and morphology (10). Altered expression of Wnt gene family proteins is associated with human carcinogenesis (7). In gastric cancer, Wnt signaling may promote self-renewal of gastric cancer stem cells (CSCs); therefore, targeting of Wnt signaling may lead to a clinical gastric cancer therapy (11). Wnt10B, localized on human chromosome 12q13 (12), was implicated in cancer development and progression by regulating nuclear factor- $\kappa \mathrm{B}$ and Notch pathways in osteosarcoma cells (13). However, Wnt10B is a bi-functional protein; one function involves $\beta$-catenin/transcription factor activation to promote tumor progression and the second function is associated with downregulating cell growth through a $\beta$-catenin-independent mechanism (14). In the present study, the association between Wnt10B mRNA and protein levels in human gastric cancer 
tissues and clinicopathological data from patients was first assessed. Wnt10B expression in regulation of gastric cancer cell proliferation and migration capacity in vitro was then investigated.

\section{Materials and methods}

Gastric cancer tissue samples. The primary gastric cancer tissues and their matched adjacent noncancerous tissues (collected $>5-10 \mathrm{~cm}$ away from the primary lesion) were obtained from 25 patients with gastric cancer who underwent surgical resection in Nanjing Medical University-Affiliated Wuxi Second Hospital (Jiangsu, China) between September 2014 and January 2015. There were 17 males and 8 females with a median age of 61 years (range, 29-75 years). All patients were histologically diagnosed with gastric adenocarcinoma and had not received any treatment prior to surgery. The present study was approved by the Ethics Committee of Nanjing Medical University-Affiliated Wuxi Second Hospital and informed consent was obtained and documented from all patients prior to involvement in the present study. Cancer tissues and adjacent noncancerous tissues were obtained and immediately stored at $-80^{\circ} \mathrm{C}$. For the present study, tissues were confirmed by pathological examination and used for reverse transcription-quantitative polymerase chain reaction (RT-qPCR) and western blotting.

Cell line and culture. The human gastric cancer SGC-7901 cell line was obtained from the Institute of Biochemistry and Cell Biology (Chinese Academy of Sciences, Shanghai, China), and maintained in Dulbecco's modified Eagle's medium (DMEM) with low-glucose (Gibco; Thermo Fisher Scientific, Inc., Waltham, MA, USA) supplemented with $10 \%$ fetal bovine serum (FBS; Gibco; Thermo Fisher Scientific, Inc.) at $37^{\circ} \mathrm{C}$ in a humidified incubator with $95 \%$ air and $5 \% \mathrm{CO}_{2}$.

Lentivirus and Wnt10B knockdown in SGC-7901 cells. A lentiviral expression vector carrying Wnt10B short hairpin RNA (shRNA) and Lenti-green fluorescent protein (GFP)-shRNA as a negative control vector were obtained from Sigma-Aldrich (EMD Millipore, Billerica, MA, USA). The Wnt10B shRNA lentiviral vector was generated by ligation of Wnt10B siRNA sequences from the Tet-pLKO-puro vector (Sigma-Aldrich; EMD Millipore). Wnt10B shRNA oligonucleotide sequences were 5'-CCGGCGGGCTCTAAG CAATGAGATTCTCGAGAATCTCATTGCTTAGAGCCCG TTTTTG-3' and 5'-AATTCAAAAACGGGCTCTAAG CAATGAGATTCTCGAGAATCTCATTGCTTAGAGCCC G-3', while the sequences of the negative control shRNA were 5'-CCGGGCAAGCTGACCCTGAAGTTCATCTCGAGATG AACTTCAGGGTCACGTTGCTTTTTG-3' and 5'-AAT TCAAAAAGCAAGCTGACCCTGAAGTTCATCTCGAGA TGAACTTCAGGGTCACGTTGC-3'. The recombinant lentivirus was produced by co-transfecting pLKO-GFP-shRNA or pLKO-Wnt10B-shRNA into HEK293T cells (Cell Bank of Type Culture Collection of the Chinese Academy of Sciences, (Beijing, China) that were previously stably transfected with packaging PU1562 and PU1563 plasmids using Lipofectamine ${ }^{\circledR}$ 2000 (Invitrogen; Thermo Fisher Scientific, Inc.), according to the manufacturer's protocol. The virus-containing supernatant was harvested at 48 and $72 \mathrm{~h}$ following transfection. Once the lentiviral titer was established, gastric cancer SGC-7901 cells were infected with multiplicity of infection of the lentivirus and selected with $1 \mu \mathrm{g} / \mathrm{ml}$ of puromycin (Invitrogen; Thermo Fisher Scientific, Inc.) for 15 days to obtain stable cell sublines. To induce shRNA expression, the cells were treated with $80 \mu \mathrm{g} / \mathrm{ml}$ doxycycline (Sigma-Aldrich: EMD Millipore) and the efficiency of Wnt10B knockdown was assessed by RT-qPCR and western blot analysis.

$R N A$ isolation and RT-qPCR. Total RNA was isolated from tissues and cells using TRIzol reagent (Invitrogen; Thermo Fisher Scientific, Inc.) and $2 \mu 1$ aliquots of RNA samples were reversely transcribed into cDNA using a HiScript 1st strand cDNA synthesis kit (Vazyme, Piscataway, NJ, USA) according to the manufacturer's protocol. The qPCR amplifications were performed using the QuantiTect SYBR-Green PCR kit (Toyobo Co., Ltd., Osaka, Japan) according to the manufacturer's protocol. Thermal cycle parameters were as follows: $95^{\circ} \mathrm{C}$ for $5 \mathrm{~min} ; 40$ cycles at $95^{\circ} \mathrm{C}$ for $30 \mathrm{sec}, 60^{\circ} \mathrm{C}$ for $30 \mathrm{sec}$, and $72^{\circ} \mathrm{C}$ for $30 \mathrm{sec}$; and $65-95^{\circ} \mathrm{C}$ drawing dissociation curve. $\beta$-actin was used as an internal control. The expression of each gene was defined from the threshold cycle $(\mathrm{Cq})$ and the melting temperatures were recorded. Using the $2^{-\Delta \Delta \mathrm{Cq}}$ method (15), relative changes in mRNA expression were analyzed. The sequences of specific primers are presented in Table I.

Protein extraction and western blot analysis. Tissues and cells were homogenized and lysed in radioimmunoprecipitation assay buffer supplemented with proteinase inhibitors $(1 \mathrm{mM}$ nphenylmethanesulfonyl fluoride; Vazyme, Piscataway, NJ, USA). The protein concentration of each sample was determined by the bicinchoninic acid protein assay kit (CWBIO, Beijing, China). Equal amounts of protein were subjected to SDS-PAGE on $12 \%$ SDS-PAGE gels and then transferred to polyvinylidene difluoride membranes. For western blot analysis, the membrane was blocked in 5\% (w/v) skim milk for $1 \mathrm{~h}$ at $37^{\circ} \mathrm{C}$ and incubated with a primary antibody for $12 \mathrm{~h}$ at $4^{\circ} \mathrm{C}$, followed by a horseradish peroxidase-conjugated anti-rabbit secondary antibody (\#AP188P; EMD Millipore) for $1 \mathrm{~h}$ at $37^{\circ} \mathrm{C}$. Protein bands were detected by the enhanced chemiluminescence detection system (GE Healthcare Life Sciences, Chalfont, UK). The primary antibodies used were: Anti-Wnt10B (dilution, 1:200; sc-25524; Santa Cruz Biotechnology, Inc., Dallas, TX, USA); GAPDH (dilution, 1:2,000; \#CW0100A; Kangcheng Pharmaceutical Co. Ltd., Guangzhou, China); Ki67 (dilution, 1:1,000; \#9449; Cell Signaling Technology, Inc., Danvers, MA, USA); $\beta$-catenin (dilution, 1:1,000; \#8480; Cell Signaling Technology, Inc.); cyclin D1 (dilution, 1:500; \#P24385; Bioworld Technology, Inc., St. Louis Park, MN, USA); N-cadherin (dilution, 1:1,000; \#13116; Cell Signaling Technology, Inc.); E-cadherin (dilution, 1:1,000; \#14472; Cell Signaling Technology, Inc.); octamer-binding transcription factor 4 (Oct4; dilution, 1:200; \#sc-365509; Santa Cruz Biotechnology, Inc.); and Nanog antibody (dilution, 1:200; \#25045; Signalway Antibody, Danvers, MA, USA).

Immunofluorescence. Tissue samples or cells were fixed in 4\% paraformaldehyde for $20 \mathrm{~min}$, permeabilized for $3 \mathrm{~min}$ 
Table I. Primer sequences for quantitative polymerase chain reaction amplification.

\begin{tabular}{lcc}
\hline Gene & \multicolumn{1}{c}{ Primer sequences } & Amplicon size, bp \\
\hline Wnt10B & & 395 \\
Forward & 5'-GCAATGGCAGCGCTCAACTC-3' \\
Reverse & ${ }^{\circ} \mathrm{C}$ \\
$\begin{array}{l}\beta \text {-actin } \\
\text { Forward }\end{array}$ & 5'-CACGAAACTACCTTCAACTCC-3' & \\
Reverse & 5'-CATACTCCTGCTTGCTGATC-3' & 265 \\
Oct4 & 5'-TTGAGGCTCTGCAGCTTAG-3' & \\
Forward & 5'-GCCGGTTACAGAACCACAC-3' & 285 \\
Reverse & & \\
Nanog & 5'-CCTGATTCTTCCACCAGTCC-3' & 292 \\
Forward & 5'-TGCTATTCTTCGGCCAGTTG-3' \\
Reverse & & \\
\hline
\end{tabular}

Oct4, octamer-binding transcription factor 4.

with $0.1 \%$ Triton $\mathrm{X}-100$, and blocked with $5 \%$ bovine serum albumin for $20 \mathrm{~min}$. The sections were then incubated with indicated aforementioned primary antibodies overnight at $4^{\circ} \mathrm{C}$, followed by incubation with $\mathrm{Cy} 3$-labeled anti-mice IgG secondary antibody at a dilution of 1:800 (\#CW0145; Kangcheng Pharmaceutical Co. Ltd.), at $37^{\circ} \mathrm{C}$ for $45 \mathrm{~min}$. The nuclei were then counterstained with DAPI (Sigma-Aldrich, EMD Millipore). The sections were reviewed under a fluorescent microscope (Nikon, Tokyo, Japan) and images were sequentially captured for evaluation.

Cell counting assay. Cells were seeded at a density of $1 \times 10^{4}$ cells per well onto 24-well plates and grown for up to 4 days. The cells were cultured at $37^{\circ} \mathrm{C}$ in humidified air containing $5 \% \mathrm{CO}_{2}$. At the end of each experiment, the cells were collected and counted in triplicate for each group at the indicated time points $(0,1,3$ and 4 days). The data were summarized as a percentage of the control.

Tumor cell wound healing assay. Cells were seeded at a density of $5 \times 10^{5}$ cells per well onto 6 -well plates and incubated for $\sim 24 \mathrm{~h}$ at $37^{\circ} \mathrm{C}$ to reach $>95 \%$ confluency of the monolayer. Wounds were then created using a sterile plastic micropipette tip on the cell monolayer and the plates were washed with PBS twice and $37^{\circ} \mathrm{C}$ cultured for an additional $24 \mathrm{~h}$. Images of the cells were captured at 0 and $24 \mathrm{~h}$ to measure the wound healing for five randomly selected fields. The width of wound healing at the original scratches was measured and calculated using NIH Image version 1.62 program (http://rsb.info.nih. gov/nih-image/). The data are expressed as the mean \pm standard deviation and presented as a percentage of the control.

Tumor cell Transwell migration assay. SGC-7901 cells were seeded at a density of $1 \times 10^{5}$ per well into the upper chamber of a $6.5 \mathrm{~mm}$ Transwell insert (\#3422; Corning Incorporated, Corning, NY, USA). DMEM supplemented with 10\% FBS was added into the bottom chamber and the cells were incubated at $37^{\circ} \mathrm{C}$ for $16 \mathrm{~h}$. At the end of the experiment, cells remaining on the membrane surface were removed with cotton swabs and cells that had migrated through the membrane (pore size, $8 \mathrm{~mm}$ ) were fixed with $4 \%$ paraformaldehyde, stained for $15 \mathrm{~min}$ at room temperature with $1 \%$ crystal violet (Sigma-Aldrich; EMD Millipore), and images were captured by light microscope (Nikon, Tokyo, Japan).

Statistical analysis. Differences in the expression of Wnt10B mRNA in paired tumor and normal tissues was analyzed by the Mann-Whitney U test with GraphPad Prism 5.0 software (GraphPad Software, La Jolla, CA, USA). Association between Wnt10B expression and clinicopathological factors was estimated by Fisher's exact test. Statistically significant correlation between two continuous variables was analyzed by the Spearman's rho test. The statistical association between two groups was analyzed by Student's t-test. $\mathrm{P}<0.05$ was considered to indicate a statistically significant difference.

\section{Results}

Upregulated Wnt10B mRNA is associated with lymph node metastasis of gastric cancer. To assess the potential role of Wnt10B in gastric cancer, RT-qPCR analysis of Wnt10B was performed in 25 pairs of gastric cancer and adjacent normal tissues. Levels of Wnt10B mRNA in gastric cancer tissues were significantly higher compared with the paired adjacent normal tissues $(\mathrm{P}<0.001$; Fig. 1A and B). Western blotting and immunofluorescence were then performed to analyze the expression of Wnt10B protein in these samples, and it was identified that levels of $\mathrm{Wnt} 10 \mathrm{~B}$ protein were also increased in gastric cancer tissues (Fig. 1C and D).

It was then determined whether associations exist between Wnt10B mRNA level and clinicopathological characteristics of patients. High levels of Wnt10B were revealed to be associated with lymph node metastasis $(\mathrm{P}=0.022$; Table II), but no associations were observed with other clinicopathological characteristics, including age, gender, tumor size, histological grade or clinical stages. 
A

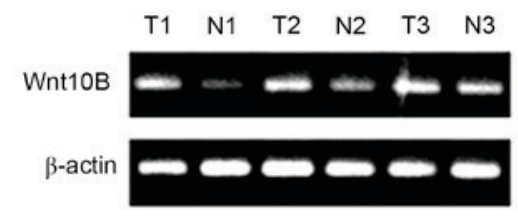

C

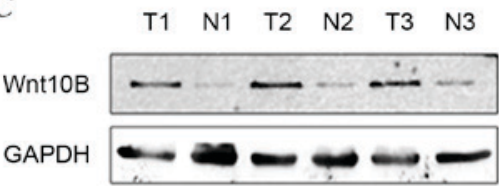

B

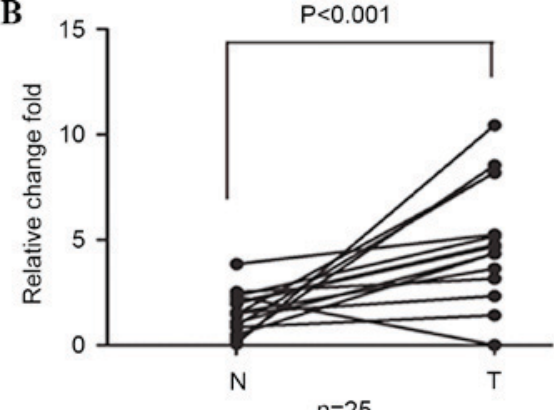

D

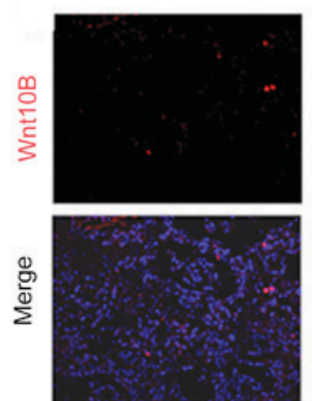

$\mathrm{N}$ $n=25$

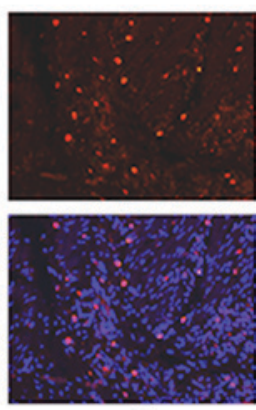

$\mathrm{T}$

Figure 1. Differential Wnt10B expression in gastric cancer and paired adjacent normal tissues. (A) Expression of Wnt10B mRNA level in gastric cancer and paired adjacent normal tissues was analyzed by semi-quantitative RT-PCR. (B) Expression of Wnt10B mRNA level in gastric cancer and paired adjacent normal tissues was analyzed by RT-qPCR. The solid line indicates matched samples. (C) Expression of Wnt10B protein level in gastric cancer and paired adjacent normal tissues was analyzed by western blot analysis. (D) Representative images of a Wnt10B immunofluorescence assay in gastric cancer and paired adjacent normal tissues. Original magnification, x200. RT-qPCR, reverse transcription-quantitative polymerase chain reaction; T, gastric cancer; N, paired adjacent normal tissues.

Wnt10B knockdown inhibits gastric cancer cell proliferation and migration in vitro. Wnt10B expression was knocked down in gastric cancer SGC-7901 cells using lentivirus carrying Wnt10B shRNA. Wnt10B shRNA significantly reduced levels of Wnt10B mRNA and protein in the Wnt10B shRNA-infected (shWnt10B) SGC-7901 cells compared with the negative control shRNA $(\mathrm{P}<0.001$; Fig. $2 \mathrm{~A}$ and $\mathrm{B})$. In addition, knockdown of Wnt10B expression inhibited gastric cancer proliferation compared with the control shRNA-infected gastric cancer cells (Fig. 2C). Ki67 immunofluorescent-staining and western blot analysis data confirmed that Wnt10B shRNA reduced tumor cell proliferation (Fig. 2D and E).

The role of Wnt10B in regulation of gastric cancer cell motility was assessed using wound healing and Transwell migration assays. Wnt10B knockdown markedly reduced gastric cancer cell wound healing and migration capacity compared with the controls (Fig. 2F and G).

Knockdown of Wnt $10 B$ expression inhibits the Wnt $/ \beta$-catenin signaling pathway and cancer cell stemness. A number of previous studies demonstrated that activation of the Wnt $/ \beta$-catenin signaling pathway was able to induce epithelial-mesenchymal transition (EMT) of cells and acquire cell stemness $(12,16-19)$. Thus, the present study confirmed the effects of Wnt10B knockdown on gastric cancer cells, and it was identified that Wnt10B knockdown reduced expression of $\beta$-catenin and the targeting gene cyclin D1 in SGC-7901 cells (Fig. 3A). The expression of the epithelial cell marker E-cadherin was upregulated, whereas expression of the mesenchymal cell marker $\mathrm{N}$-cadherin was downregulated (Fig. 3A and B), indicating a reversed EMT phenomenon in gastric cancer cells. Subsequently, expression of the stem cell markers Oct4 and Nanog was detected in the Wnt10B knocked down cells. Expression of Oct4 and Nanog proteins was decreased in Wnt10B knocked down cells compared with the control cells (Fig. 3C). Therefore, it was speculated that Wnt10B may increase the number of stem cells in gastric cancer tissue. Thus, the Wnt10B mRNA and stem cell markers Oct4 and Nanog mRNA were detected in gastric cancer tissues by real-time RT-PCR, and the association between Wnt10B, Oct4 and Nanog was analyzed. The results demonstrated that there was a positive correlation between Wnt10B and Oct4 $(\mathrm{r}=0.8033)$, and Wnt10B and Nanog $(\mathrm{r}=0.7630)$ in gastric cancer tissues (Fig. 3D).

\section{Discussion}

One hallmark of tumor biology is activation of the canonical Wnt pathway and upregulated stability and nuclear localization of $\beta$-catenin to promote expression of targeted genes during human tumorigenesis or development (20-22). Upregulation of Wnt/B-catenin signaling can enhance EMT in different types of cancer cells, including prostate, hepatocellular and gastric cancer cells (16-19), as well as proliferation and formation of CSCs $(11,23)$. The present study first analyzed the association of Wnt10B expression in human gastric cancer tissues with clinicopathological data from patients, and then investigated the role of Wnt10B in the regulation of gastric cancer cell proliferation and migration, and the underlying molecular events in vitro. The present data demonstrated that Wnt10B mRNA and protein were upregulated in gastric cancer tissues compared with the paired normal tissues. The upregulated 
Table II. Association of Wnt10B expression with clinicopathological factors from patients with gastric cancer.

\begin{tabular}{|c|c|c|c|c|}
\hline Factor & Number $(\%)$ & High Wnt10B, n & Low Wnt10B, n & P-value \\
\hline Age & & & & 0.63 \\
\hline$\leq 60$ years & $10(40)$ & 8 & 2 & \\
\hline$>60$ years & $15(60)$ & 10 & 5 & \\
\hline Gender & & & & 0.60 \\
\hline Male & $17(68)$ & 12 & 5 & \\
\hline Female & $8(32)$ & 6 & 2 & \\
\hline Size & & & & 0.63 \\
\hline$\leq 5 \mathrm{~cm}$ & $19(76)$ & 10 & 9 & \\
\hline$>5 \mathrm{~cm}$ & $6(24)$ & 3 & 3 & \\
\hline Stage & & & & 0.60 \\
\hline $\mathrm{I} / \mathrm{II}$ & $18(72)$ & 11 & 7 & \\
\hline III/IV & $7(28)$ & 4 & 3 & \\
\hline Histological grade & & & & 0.45 \\
\hline $\mathrm{W} / \mathrm{M}$ & $7(28)$ & 3 & 4 & \\
\hline Poorly/signet & $18(72)$ & 10 & 8 & \\
\hline Tumor grade & & & & 0.54 \\
\hline $\mathrm{T} 1 / \mathrm{T} 2$ & $19(76)$ & 11 & 8 & \\
\hline $\mathrm{T} 3 / \mathrm{T} 4$ & $6(24)$ & 4 & 2 & \\
\hline Lymph node metastasis & & & & 0.022 \\
\hline Present & $20(80)$ & 20 & 0 & \\
\hline Absent & $5(20)$ & 2 & 3 & \\
\hline
\end{tabular}

Wnt10B expression was associated with gastric cancer metastasizing to lymph nodes. In addition, knockdown of Wnt10B expression reduced gastric cancer cell proliferation and migration and expression of Ki67 protein. Knockdown of Wnt10B expression also inhibited tumor cell EMT by upregulating E-cadherin and downregulating $\mathrm{N}$-cadherin and tumor cell stemness, via downregulation of Oct4 and Nanog expression. The data from the present study indicated that knockdown of Wnt10B expression may have a role in suppressing gastric cancer progression. Additional studies are required to investigate whether targeting Wnt10B expression or activity may be a novel strategy to control gastric cancer.

Wnt10B, a member of the Wnt family of proteins (24), is conserved among diverse species and serves a crucial role in normal tissue development $(25,26)$. A previous study revealed that Wnt10B activates canonical $\beta$-catenin signaling to promote development of breast cancer (27). It was also suggested that Wnt10B is expressed differently in benign and malignant diseases, and has the potential to be a tumor biomarker in molecular diagnosis of human cancer (28). In the present study, the usefulness of detection of Wnt10B expression to differentiate normal tissues from tumor tissues was confirmed. In addition, the present data also revealed that Wnt10B expression is associated with gastric cancer lymph node metastasis, indicating that Wnt10B is not only altered early in gastric cancer development, but is also associated with gastric cancer progression. Additional studies with a larger sample size are required to confirm the present data.

Furthermore, the present data demonstrated that knockdown of Wnt10B expression inhibits proliferation and migration of gastric cancer cells. It is widely accepted that cancer metastasis is one of the malignant properties of cancer and the main cause of cancer-associated mortality (29). Wnt10B-associated tumor cell migration and wound healing may promote gastric cancer progression. Phenotypically, tumor cell EMT is an important process in cancer development and metastasis (30). When cancer cells acquire EMT phenotypes, they invade the surrounding tissue and eventually metastasize to distant sites (31). The present study showed that knockdown of Wnt10B inhibits migration of gastric cancer cells via suppression of tumor cell EMT. This is evident by enhanced expression of the epithelial marker E-cadherin, but reduced expression of mesenchymal marker $\mathrm{N}$-cadherin in gastric cancer cells.

In addition, CSCs have been identified in a variety of human cancers, such as breast, brain, prostate, melanoma, colon, liver, pancreatic and head and neck cancer, and are able to initiate tumorigenesis and cause tumor metastasis (32). A previous study demonstrated that activation of the $\mathrm{Wnt} / \beta$-catenin pathway in cancer cells induces their stem cell characteristics (33). The stem cell markers Oct4, sex-determining region Y-box 2, Nanog and Krüppel-like factor 4 are important target genes of the Wnt/ $\beta$-catenin pathway (34). In the present study, it was identified that Wnt10B level was positively associated with stem cell markers in gastric cancer tissues, supporting that Wnt10B may be involved in the generation and maintenance of CSCs in gastric cancer. These data were also confirmed in Wnt10B knockdown cells, demonstrating that reduced Wnt10B expression also downregulated expression of Oct4 and Nanog in gastric cancer cells in vitro. Further studies 


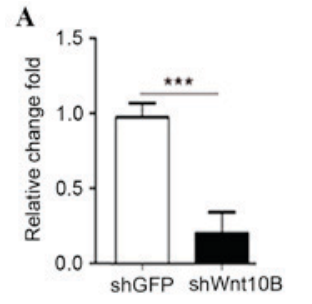

D

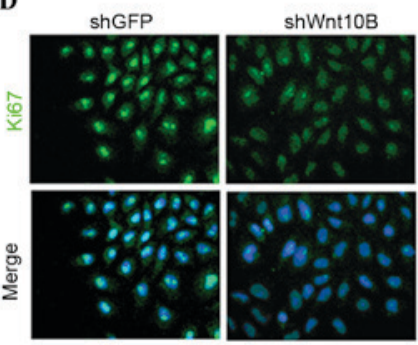

$\mathbf{F}$

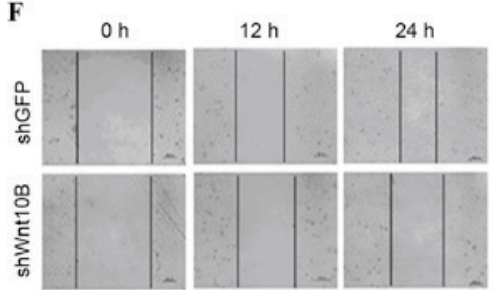

B

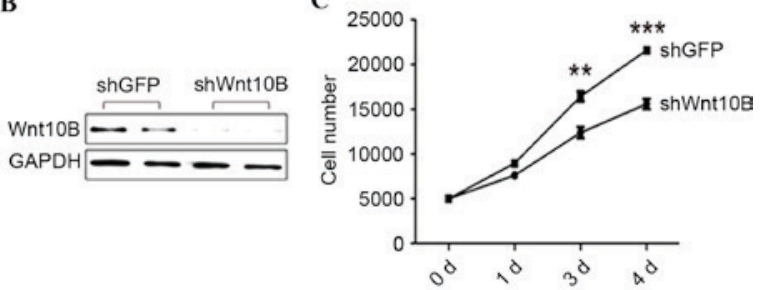

$\mathbf{E}$
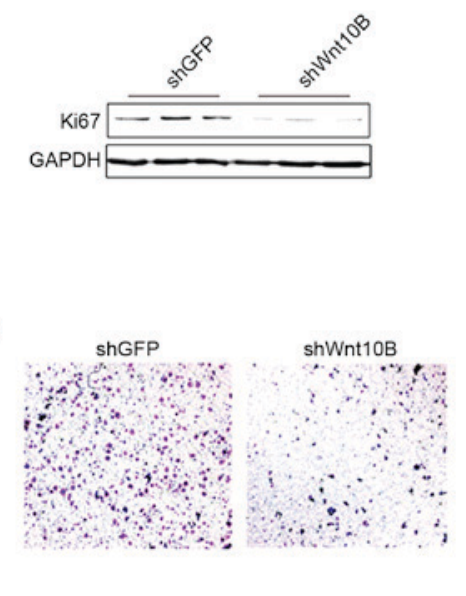

Figure 2. Effects of Wnt10B knockdown on the inhibition of gastric cancer cell proliferation and migration. (A) SGC-7901 cells were infected with lentivirus carrying Wnt10B shRNA or negative control shRNA and expression of Wnt10B mRNA was analyzed by RT-qPCR. (B) Duplicated cells were subjected to western blot analysis of Wnt10B protein expression. (C) Cell counting assay. Growth curves of Wnt10B-knockdown SGC-7901 cells (shWnt10B) and control cells (shGFP) are presented as the mean \pm standard deviation and plotted in the graph. (D) Immunofluorescence analyses of Ki67 expression in Wnt10B knocked down SGC-7901 (shWnt10B) and control cells (shGFP). Original magnification, x400. (E) Western blot analyses of Ki67 expression in Wnt10B-knockdown SGC-7901 (shWnt10B) and control cells (shGFP). (F) Wound healing assay and (G) Transwell migration assay. SGC-7901 cells were infected with lentivirus carrying Wnt10B shRNA or negative control shRNA and subjected to wound healing and Transwell migration assays. Original magnification, $\mathrm{x} 100$. ${ }^{* *} \mathrm{P}<0.01$ and ${ }^{* * *} \mathrm{P}<0.001$, compared with control cells. GFP, green fluorescent protein; shRNA, short hairpin RNA; RT-qPCR, reverse transcription polymerase chain reaction.

$\mathbf{A}$

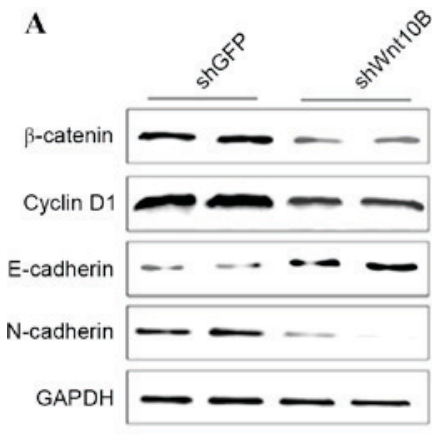

C

\section{1}

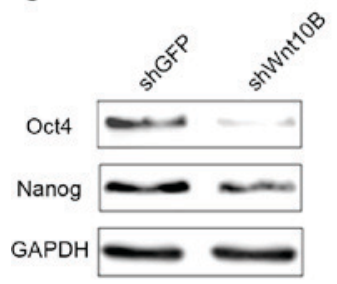

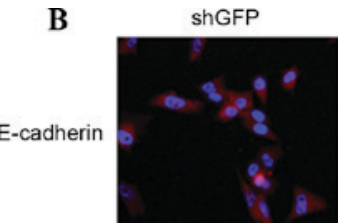
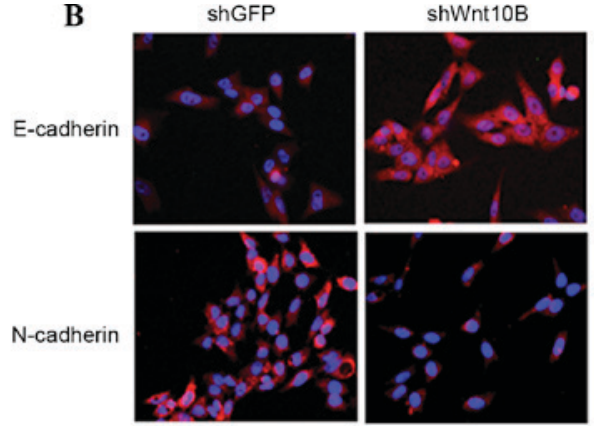
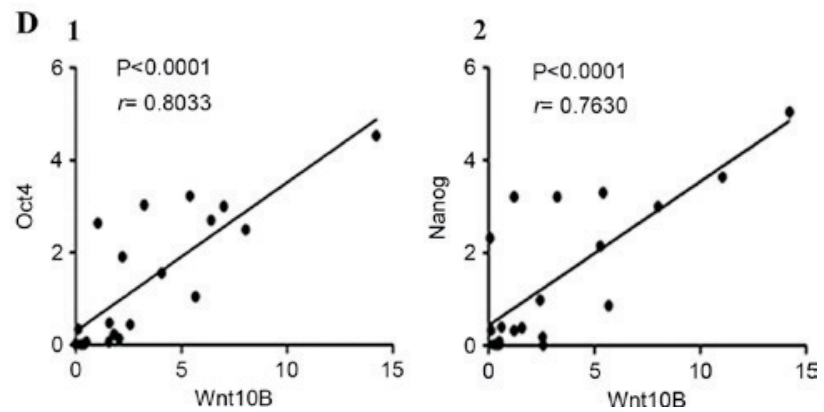

Figure 3. Effects of Wnt10B knockdown on the regulation of gene expression. (A) Western blot analysis of $\beta$-catenin, cyclin D1, E-cadherin and N-cadherin expression in Wnt10B knocked down SGC-7901 (shWnt10B) and negative control cells (shGFP). (B) Immunofluorescence analyses of E-cadherin and $\mathrm{N}$-cadherin expression in Wnt10B knocked down SGC-7901 (shWnt10B) and negative control cells (shGFP). Original magnification, x400. (C) Western blot analysis of stem cell markers Oct4 and Nanog expression in Wnt10B knocked down SGC-7901 (shWnt10B) and negative control cells (shGFP). (D) Correlation between Wnt10B and Oct4 and Nanog mRNA levels in gastric cancer tissues. There was a positive correlation between mRNA levels of expression. Oct4, octamer-binding transcription factor 4; shRNA, short hairpin RNA; GFP, green fluorescent protein. 
may confirm whether Wnt10B knockdown inhibits $\beta$-catenin translocation into cell nuclei, as shown by previous studies in other cancers (35-38).

The present study provides proof-of-principle, demonstrating that alteration in Wnt10B expression is associated with gastric cancer. Future studies may confirm the effects of Wnt10B in gastric cancer to provide a novel diagnostic and prognostic biomarker for gastric cancer and a therapeutic strategy to control gastric cancer. In addition, the mechanisms responsible for the aberrant expression of Wnt10B in gastric cancer will also be investigated. Furthermore, the authors will perform animal experiments to verify the effects of Wnt10B knockdown in suppression of gastric cancer metastasis.

\section{Acknowledgements}

The present study was supported in part by a grant from the National Natural Science Foundation of China (grant no. 81301503).

\section{References}

1. Tan YK and Fielding JW: Early diagnosis of early gastric cancer Eur J Gastroenterol Hepatol 18: 821-829, 2006.

2. Ding YB, Xia TS, Wu JD, Chen GY, Wang S and Xia JG: Surgical outcomes for gastric cancer of a single institute in southeast China. Am J Surg 203: 217-221, 2012.

3. Lee YY and Derakhshan MH: Environmental and lifestyle risk factors of gastric cancer. Arch Iran Med 16: 358-365, 2013.

4. González CA, Sala N and Rokkas T: Gastric cancer: Epidemiologic aspects. Helicobacter 18 (Suppl 1): S34-S38, 2013

5. World Cancer Report 2014. World Health Organization. Chapter 5.4, 2014.

6. Wang M, Gu H, Qian H, Zhu W, Zhao C, Zhang X, Tao Y, Zhang $\mathrm{L}$ and $\mathrm{Xu}$ W: miR-17-5p/20a are important markers for gastric cancer and murine double minute 2 participates in their functional regulation. Eur J Cancer 49: 2010-2021, 2013.

7. Nusse R and Varmus HE: Many tumors induced by the mouse mammary tumor virus contain a provirus integrated in the same region of the host genome. Cell 31: 99-109, 1982.

8. Chen H, Wang Y and Xue F: Expression and the clinical significance of Wnt10a and Wnt10b in endometrial cancer are associated with the Wnt/ß-catenin pathway. Oncol Rep 29: 507-514, 2013.

9. Cadigan KM and Liu YI: Wnt signaling: Complexity at the surface. J Cell Sci 119: 395-402, 2006.

10. Kohn AD and Moon RT: Wnt and calcium signaling: Beta-catenin independent pathways. Cell Calcium 38: 439-446, 2005.

11. Mao J, Fan S, Ma W, Fan P, Wang B, Zhang J, Wang H, Tang B, Zhang Q, Yu X, et al: Roles of Wnt/ $\beta$-catenin signaling in the gastric cancer stem cells proliferation and salinomycin treatment. Cell Death Dis 5: e1039, 2014.

12. Katoh M: $W N T$ and $F G F$ gene clusters (Review). Int J Oncol 21 1269-1273, 2002.

13. Mödder UI, Oursler MJ, Khosla S and Monroe DG: Wnt10b activates the Wnt, notch, and $\mathrm{NF \kappa B}$ pathways in U2OS osteosarcoma cells. J Cell Biochem 112: 1392-1402, 2011.

14. Yoshikawa H, Matsubara K, Zhou X, Okamura S, Kubo T, Murase Y, Shikauchi Y, Esteller M, Herman JG, Wei Wang $X$ and Harris CC: WNT10B functional dualism: Beta-catenin/Tcf-dependent growth promotion or independent suppression with deregulated expression in cancer. Mol Biol Cell 18: 4292-4303, 2007.

15. Livak KJ and Schmittgen TD. Analysis of relative gene expression data using real-time quantitative PCR and the 2(-Delta Delta C(T)) Method. Methods 25: 402-408, 2001.

16. Liu H, Yin J, Wang H, Jiang G, Deng M, Zhang G, Bu X, Cai S, Du J and He Z: FOXO3a modulates WNT/ $\beta$-catenin signaling and suppresses epithelial-to-mesenchymal transition in prostate cancer cells. Cell Signal 27: 510-518, 2015.
17. Jiang L, Yang YD, Fu L, Xu W, Liu D, Liang Q, Zhang X, Xu L, Guan XY, Wu B, et al: CLDN3 inhibits cancer aggressiveness via Wnt-EMT signaling and is a potential prognostic biomarker for hepatocellular carcinoma. Oncotarget 5: 7663-7676, 2014.

18. Cong N, Du P, Zhang A, Shen F, Su J, Pu P, Wang T, Zjang J, Kang $\mathrm{C}$ and Zhang Q: Downregulated microRNA-200a promotes EMT and tumor growth through the wnt/ $\beta$-catenin pathway by targeting the E-cadherin repressors ZEB1/ZEB2 in gastric adenocarcinoma. Oncol Rep 29: 1579-1587, 2013.

19. Voon DC, Wang H, Koo JK, Nguyen TA, Hor YT, Chu YS, Ito K, Fukamachi H, Chan SL, Thiery JP and Ito Y: Runx3 protects gastric epithelial cells against epithelial-mesenchymal transition-induced cellular plasticity and tumorigenicity. Stem Cell 30: 2088-2099, 2012.

20. Brown AM: Canonical Wnt signaling: High-throughput RNAi widens the path. Genome Biol 6: 231, 2005.

21. Li C, Song G, Zhang S, Wang E and Cui Z: Wnt3a increases the metastatic potential of non-small cell lung cancer cells in vitro in part via its upregulation of Notch3. Oncol Rep 33: 1207-1214, 2015.

22. Qi L, Sun B, Liu Z, Cheng R, Li Y and Zhao X: Wnt3a expression is associated with epithelial-mesenchymal transition and promotes colon cancer progression. J Exp Clin Cancer Res 33: 107,2014

23. Shojima K, Sato A, Hanaki H, Tsujimoto I, Nakamura M, Hattori K, Sato Y, Dohi K, Hirata M, Yamamoto H and Kikuchi A: Wnt5a promotes cancer cell invasion and proliferation by receptor-mediated endocytosis-dependent and -independent mechanisms, respectively. Sci Rep 5: 8042, 2015.

24. Lee FS, Lane TF, Kuo A, Shackleford GM and Leder P: Insertional mutagenesis identities a member of the Wnt gene family as a candidate oncogene in the mammary epithelium of int-2/Fgf-3 transgenic mice. Proc Natl Acad Sci USA 92: 2268-2272, 1995

25. Nusse R and Varmus HE: Wnt genes. Cell 69: 1073-1087, 1992.

26. Moon RT, Brown JD and Torres M: WNTs modulate cell fate and behavior during vertebrate development. Trends Genet 13: 157-162, 1997.

27. Wend P, Runke S, Wend K, Anchondo B, Yesayan M, Jardon M, Hardie N, Loddenkemper C, Ulasov I, Lesniak MS, et al: WNT10B/ $\beta$-catenin signalling induces HMGA2 and proliferation in metastatic triple-negative breast cancer. EMBO Mol Med 5: 264-279, 2013.

28. Bui TD, Rankin J, Smith K, Huguet EL, Ruben S, Strachan T, Harris AL and Lindsay S: A novel human gene, WNT10B, maps to $12 \mathrm{q} 13$ and is expressed in human breast carcinomas. Oncogene 14: 1249-1253, 1997.

29. Guan X: Cancer metastases: Challenges and opportunities. Acta Pharm Sin B 5: 402-418, 2015.

30. Ye X and Weinberg RA: Epithelial-mesenchymal plasticity: A central regulator of cancer progression. Trends Cell Biol 25: 675-686, 2015.

31. Lee JM, Dedhar S, Kalluri R and Thompson EW: The epithelial-mesenchymal transition: New insights in signaling, development, and disease. J Cell Biol 172: 973-981, 2006.

32. Takaishi S, Okumura T and Wang TC: Gastric cancer stem cells. J Clin Oncol 26: 2876-2882, 2008.

33. Arend RC, Londoño-Joshi AI, Straughn JM Jr and Buchsbaum DJ: The Wnt/ $\beta$-catenin pathway in ovarian cancer: A review. Gynecol Oncol 131: 772-779, 2013.

34. Holland JD, Klaus A, Garratt AN and Birchmeier W: Wnt signaling in stem and cancer stem cells. Curr Opin Cell Biol 25: 254-264, 2013.

35. Bao Z, Wang Y, Yang L, Wang L, Zhu L, Ban N, Fan S, Chen W, Sun J, Shen C and Cui G: Nucleostemin promotes the proliferation of human glioma via Wnt/ $\beta$-Catenin pathway. Neuropathology 36: 237-249, 2016.

36. Wangpu X, Yang X, Zhao J, Lu J, Guan S, Lu J, Kovacevic Z, Liu W, Mi L, Jin R, et al: The metastasis suppressor, NDRG1, inhibits 'stemness' of colorectal cancer via down-regulation of nuclear $\beta$-catenin and CD44. Oncotarget 6: 33893-33911, 2015.

37. Guo Q and Qin W: DKK3 blocked translocation of $\beta$-catenin/EMT induced by hypoxia and improved gemcitabine therapeutic effect in pancreatic cancer Bxpc-3 cell. J Cell Mol Med 19: 2832-2841, 2015.

38. Burkhalter RJ, Westfall SD, Liu Y and Stack MS: Lysophosphatidic acid initiates epithelial to mesenchymal transition and induces $\beta$-catenin-mediated transcription in epithelial ovarian carcinoma. J Biol Chem 290: 22143-22154, 2015. 